\title{
Index rerum ad Vol 176
}

\section{Confecit: C. Loeb-Schürch}

$(\mathrm{V})=$ Proceedings - Verhandlungsberichte -

Congrès $(\mathrm{B})=$ Book Reviews - Buchbesprechungen -

Livres nouveaux

AAR, v. Immunopathology

Ablatio, v. Aphakie, Fibroplasie

Ablation treatment; indications for 'ablation

treatment' of diabetic retinopathy 271 (V) Acid maltase deficiency, v. Glycogenosis Accident, v. Silikonimplantate Acromegaly; diabetic retinopathy in

acromegaly 74 Actinomycosis, v. Dacryocystitis Acuité visuelle, v. Keratoconus Adaptation à la lumière, v. Eye dipoles Aderhaut, v. Heredodystrophies, Siderosis Adverse effects of drugs used in the treatment

of glaucoma $264(\mathrm{~V})$ Aging of the crystalline lens and changes of protein composition 281 (V) $\alpha$-L-iduronidase, v. Hurler/Scheie phenotype Amblyopia, v. Fibroplasie Amyloidosis; fluorographical findings in familial primary amyloidosis 301 Anastomose, v. Congenital malformations Angiographie, v. Amyloidosis, Electrophysiological studies,

Irisangiographie, Rétinopathie diabétique Angle camérulaire, v. Irisangiographie,

Neurofibromatosis, Trabeculotomy Aniridia, cataract and gonadoblastoma in a mentally retarded girl with deletion of chromosome 11. A clinicopathological case

report 171 Anterior chamber, v. Siderosis Antibiotics; complications of antibiotic therapy 263 (V) Anticorps, v. Immunopathology A-patterns; desinsertion of the superior oblique in A-patterns 287 (V) Aphakie; les lesions rétiniennes périphériques

dans Taphakie 12

Aqueous humour, v. Trabeculotomy Areflexie, v. Polyneuritis

Arterial hypertension, v. Rétinopathie diabétique Artérite à cellules géantes, v. Ischaemie Astrocytoma; pilocytic (juvenile) astrocytoma of

the optic nerve. The influence of biopsy 181 Ataxie, v. Polyneuritis Augeninnendruck, v.

Komplikationen Augenkomplikationen, v. Astrocytoma,

Komplikationen Augenmuskeln, v. Polyneuritis, Silikonimplantate,

Sutures

Basaliom, v. Erythrodermia ichthyosiformis Bindehaut, v. Hurler/Scheie phenotype Biochemie, v. Glycogenosis, Hurler/Scheie phenotype, Immunopathology Biométrie oculaire clinique (oculométrie) 120 (B)

Biomicroscopie, v. Aphakie

Biomicroscopy of the corneal endothelium 274 (V) Biopsy, v. Astrocytoma Blicklähmung, v.

Polyneuritis Bone, v. Osteo-Odonto-Keratoplastik, 
Silikonimplantate Book reviews 59 (B), 120 (B), 178 (B) Buchbesprechungen 59 (B), 120 (B), 178 (B) Buphthalmus, v. Neurofibromatosis

'Cartwheel maculopathy' 272 (V) Cataract, v. Aniridia, Immunopathology,

Komplikationen, Sutures Cataract and lens; pathological-optic approach

of cataract and lens 331 Cell inclusions, v. Fabry's disease, Glycogenosis,

Siderosis Central vein occlusion, v. Irisangiographie,

Retinal branch vein occlusion Céramiques de verre, v. Osteo-Odonto-Keratoplastik Chamber angle, v. Irisangiographie,

Neurofibromatosis, Trabeculotomy Chambre antérieure, v. Siderosis Chiasma, v. Astrocytoma Chien, v. Osteo-Odonto-Keratoplastik China; a glimpse of ophthalmology in China

$1976286(\mathrm{~V})$

Index rerum ad Vol. 176

351

Chirurgie, v. Astrocytoma, Fibroplasie, Greffon

cornéen, Komplikationen, Silikonimplantate,

Sutures, Tarsal plates Chirurgie maxillaire, v. Silikonimplantate Chordom der Orbita 236

Chorioidea, v. Heredodystrophies, Siderosis Chromosom, v. Aniridia, Hurler/Scheie phenotype

Ciliary body, v. Siderosis Circulation, v. Irisangiographie Classification, v. Heredodystrophies

Clinicopathologic case reports 53, 171, 236 Collagenase; some effects of collagenase on the crystalline lens. A preliminary

communication 283 (V) Complement, v. Immunopathology Complications oculaires, v.

Astrocytoma,

Komplikationen Congenital malformations of the retinal vessels

with primary optic nerve involvement 86 Congrès 262-300 (V) Conjunctiva, v. Hurler/Scheie

phenotype Contact lenses, v. Keratoconus Contraceptives; contact lens tolerance and the

use of oral contraceptives 288 (V) Cornea, v. Fabry’s disease, Greffon cornéen,

Hurler/Scheie phenotype, Keratitis sicca,

Keratoconus, Komplikationen,

Osteo-Odonto-Keratoplastik, Rejection Corneal graft, v. Greffon cornéen,

Komplikationen, Osteo-Odonto-Keratoplastik,

Rejection Corneal transplants; first experience with

HLA-matched corneal transplants in high

risk cases 275 (V) Corps ciliaire, v. Siderosis Corps étranger, v. Siderosis Corps vitré, v.

Amyloidosis, Aphakie,

Fibroplasie, Siderosis Cristallin, v. Aniridia, Cataract and lens,

Immunopathology, Siderosis Cryothérapie, v. Lidtumoren Cytologie, v. Keratitis sicca

DA, v. Eye dipoles, Tilted disc

Dacryocystitis; actinomycotic dacryocystitis 145

Deafness, v. Aniridia

Décollement rétinien, v. Aphakie, Fibroplasie

Decompression; results of transantral

decompression in hyperthyroid

exophthalmos 291 (V)

Decompression; surgical decompression of the 
orbit 278 (V) Dégénérescence rétinienne, v. Aphakie Dermatologie, v. Erythrodermia ichthyosiformis Development; the effects of blindness and other impairments on early development 178 (B) Dexon, v. Sutures Diabetes, v. Cataract and lens, Irisangiographie,

Polyneuritis Diabetic retinopathy, v. Acromegaly,

Electrophysiological studies, Rétinopathie

diabétique Diplopia, v. Plasmacytoma, Silikonimplantate Disc, v. Ischaemie, Tilted disc Dog, v. Osteo-Odonto-Keratoplastik Drug treatment of diabetic retinopathy:

possibilities, drawbacks and dangers 262 (V) Dyschromatopsia, v. Vitelline dystrophy Dyslexia: a perceptual chaos 296 (V) Dystrophie maculaire, v. Vitelline dystrophy Dystrophies tapétorétiniennes,

v. Heredodystrophies, Hurler/Scheie

phenotype

EEG, v. Eye dipoles Electro-ophthalmology and the diagnosis of

adverse effects of drugs 265 (V) Electrophysiological studies before and after

argon-laser photocoagulation in diabetic

retinopathy 133 Electrophysiology, v. Eye dipoles, Vitelline

dystrophy Elektronenmikroskopie, v. Astrocytoma,

Fabry's disease, Glycogenosis, Human retina,

Hurler/Scheie phenotype, Mitosis Endocrine exophthalmos 277 (V) Endocrinology, v.

Acromegaly Endothel, v. Greffon cornéen Enzym, v. Fabry's disease, Glycogenosis,

Hurler/Scheie phenotype EOG, v. Electrophysiological studies,

Eye dipoles, Tilted disc, Vitelline dystrophy Epithelium pigmentaire, v. Heredodystrophies,

Siderosis, Vitelline dystrophy ERG, v. Electrophysiological studies,

Hurler/Scheie phenotype, Siderosis, Tilted disc Erythrodermia ichthyosiformis; aggressives

Lidbasaliom bei Erythrodermia ichthyosiformis

congenita sicca 53 Exfoliation; the exfoliation syndrome 295 (V)

352

Index rerum ad Vol. 176

Exophthalmos, v. Chordom, Decompression,

Plasmacytoma Experimentelle Forschung, v. Immunopathology,

Keratitis sicca, Lidtumoren, Osteo-Odonto-

Keratoplastik, Rejection, Trabeculotomy Extramedulläres Plasmozytom, v. Plasmacytoma Eye

dipoles; current views and some suggestions

about mutual influences of eye dipoles as

revealed by EOG 343

Fabry's disease; corneal ultrastructural changes

in Fabry's disease 313 Fibroplasie; klinisches Bild und Prognose der Ab-

latio retinae bei retrolentaler Fibroplasie 252 Fisher-Syndrom, v. Polyneuritis Fluoro-

Angiographie, v. Amyloidosis,

Electrophysiological studies, Irisangiographie,

Rétinopathie diabétique Foreign body, v. Siderosis Fraktur, v. Silikonimplantate Fremdkörper, v.

Siderosis Frühgeburt, v. Fibroplasie

Gefässe, v. Acromegaly, Amyloidosis,

Congenital malformations, Electrophysiological 
studies, Fabry's disease, Irisangiographie,

Ischaemie, Rétinopathie diabétique Gefässneubildung, v. Irisangiographie,

Rétinopathie diabétique Geistesschwäche, v. Aniridia Genetics, v. Erythrodermia

ichthyosiformis,

Fabry's disease, Heredodystrophies,

Hurler/Scheie phenotype, Neurofibromatosis,

Tilted disc, Vitelline dystrophy Giant cell arteritis, v. Ischaemie Glaskeramik, v. Osteo-Odonto-

Keratoplastik Glaskörper, v. Amyloidosis, Aphakie,

Fibroplasie, Siderosis Glaucoma, v. Irisangiographie, Neurofibromatosis,

Trabeculotomy Glaucoma; Ophthalmology series: clinical

glaucoma 178 (B) Glaucomas; Becker-Shaffer's diagnosis and

therapy of the glaucomas 179 (B) Glaucome à angle fermé, v. Irisangiographie Glycogenosis;

ultrastructural observations on the

retina in type II glycogenosis

(Pompe's disease) 61 Glycolipids, v. Fabry's disease Gonadoblastoma, v. Aniridia

Graft, v. Greffon cornéen, Osteo-Odonto-Keratoplastik, Silikonimplantate, Tarsal plates

Greffe, v. Greffon cornéen, Komplikationen, Osteo-Odonto-Keratoplastik, Rej ection,

Silikonimplantate, Tarsal plates

Greffon cornéen; importance de la viabilité de Гendothélium du greffon cornéen 245

Growth hormone, v. Acromegaly

Haemangioma racemosum, v. Congenital malformations

Hallermann-Streiff; the Hallermann-Streiff syndrome 280 (V)

Hautkrankheiten, v. Eryhtrodermia ichthyosiformis

Hemeralopie, v. Tilted disc

Hémorragie rétinienne, v. Aphakie

Hérédité, v. Erythrodermia ichthyosiformis, Fabry's disease, Heredodystrophies, Hurler/Scheie phenotype, Neurofibromatosis, Tilted disc, Vitelline dystrophy

Heredodystrophies; classification of the diffuse chorioretinal heredodystrophies or peripheral

tapetoretinal dystrophies 1

Histochemie, v. Keratitis sicca

Histologie, v. Aniridia, Astrocytoma, Chordom, Erythrodermia ichthyosiformis, Fabry's disease,

Greffon cornéen, Hurler/Scheie phenotype, Keratitis sicca, Lidtumoren, Mitosis,

Neurofibromatosis, Plasmacytoma, Rejection, Retinoblastoma, Siderosis

Histoplasmosis; presumed histoplasmosis 273 (V)

Homocystinuria $282(\mathrm{~V})$

Hormonspiegel, v. Acromegaly

Hornhaut, v. Fabry's disease, Greffon cornéen, Hurler/Scheie phenotype, Keratitis sicca,

Keratoconus, Komplikationen, Osteo-Odonto-Keratoplastik, Rejection

Human retina; scanning electron microscopic study of the human retina 308

Humeur aqueuse, v. Trabeculotomy

Hund, v. Osteo-Odonto-Keratoplastik

Hurler/Scheie phenotype. Report of an inbred sibship with tapeto-retinal degeneration and

electron-microscopic examination of the conjunctiva 194

Hypermetropia, v. Cataract and lens

Hypertonie, v. Rétinopathie diabétique

Hypoplasia nervi optici, v. Congenital malformations 
Hypoxie, v. Irisangiographie

Index rerum ad Vol. 176

353

Ichthyosis, v. Erythrodermia ichthyosiformis Immunodepression, v. Greffon corneen, Rejection Immunopathology of the lens. I. 86Rb efflux and

protein leakage from normal lenses exposed

to unrelated antigen-antibody interactions 164 Immunopathology of the lens. II. s $\beta R$ b efflux and protein leakage from normal lenses exposed

to antilens and antiuveoretina antibodies 230 Immunopathology of the lens. i $\prod$. The effect of experimental allergic uveitis on the cation

balance in the lens 258 Instruments; evaluation of a number of

ophthalmological instruments 281 (V) Interocular influences, v. Eye dipoles Ionen, v.

Immunopathology, Siderosis Iridocapsular lenses; the mechanism of fixation

of iridocapsular lenses 285 (V) Iris, v. Aniridia, Siderosis Irisangiographie bei Rubeosis iridis 91

Irish Ophthalmological Society, v. Netherlands

Ophthalmological Society Ischaemie; akute Ischaemie des Nervus opticus 69

lumeaux, v. Retinoblastoma

Kammerwasser, v. Trabeculotomy Kammerwinkel, v. Irisangiographie,

Neurofibromatosis, Trabeculotomy Kaninchen, v. Immunopathology, Keratitis sicca,

Lidtumoren, Osteo-Odonto-Keratoplastik,

Rejection Katarakt, v. Aniridia, Immunopathology,

Komplikationen, Sutures Keratitis sicca; cytology of the superficial

keratinised cells in experimental keratitis

sicca 113 Keratoconus. A disease that can be treated with

soft lenses 34 Keratoplastik, v. Greffon corneen, Komplikationen,

Osteo-Odonto-Keratoplastik, Rejection Kieferchirurgie, v. Silikonimplantate Klassierung, v.

Heredodystrophies Knochen, v. Osteo-Odonto-Keratoplastik,

Silikonimplantate Komplement, v. Immunopathology Komplikationen der kombinierten

Katarakt-

Keratoplastik-Operation 39 Kontaktglas, v. Aphakie Kontaktlinsen, v. Keratoconus

Kryotherapie, v. Lidtumoren

Lacrimal gland; enigmatic lacrimal gland

tumours 279 (V) Lacrimal pathways, v. Dacryocystitis,

Lidtumoren Lähmung, v. Polyneuritis, Silikonimplantate Lapin, v. Immunopathology, Keratitis

sicca,

Lidtumoren, Osteo-Odonto-Keratoplastik,

Rejection Laserkoagulation, v. Electrophysiological studies,

Irisangiographie, Trabeculotomy Lens, v. Aniridia, Cataract and lens,

Immunopathology, Siderosis Lens implantation after extracapsular lens

extraction 284 (V) Lens implantation; ocular pathology after

implantation of artificial lenses 285 (V) Lens implantation; the drawback of lens

implantation 285 (V) Lentilles de contact, v. Keratoconus Lichtadaptation, v. Eye dipoles Lid, v. Erythrodermia ichthyosiformis,

Lidtumoren, Tarsal plates Lidtumoren; das Verhalten der Tränenwege nach

Kryotherapie von Lidtumoren 150 Lightcoagulation; diabetic retinopathy and 
lightcoagulation 270 (V) Linse, v. Aniridia, Cataract and lens, Immunopathology, Siderosis Lipid metabolism, v. Fabry's disease Liquor cerebro-spinalis, v. Polyneuritis Livres nouveaux 59 (B), 120 (B), 178 (B) Lysosome, v. Fabry's disease, Glycogenosis

Macrophage, v. Siderosis Macula, v. Vitelline dystrophy Malformation, v. Aniridia, Congenital malformations, Neurofibromatosis,

Retinoblastoma Maxillary surgery, v. Silikonimplantate Melanoma; dimensions of the malignant melanoma of the choroid 266 (V) Membran-Permeabilität, v. Immunopathology Meningioma, v. Astrocytoma Mental retardation, v. Aniridia Metabolism, v. Fabry's disease, Glycogenosis, Heredodystrophies, Hurler/Scheie phenotype,

Irisangiographie, Vitelline dystrophy Methode, v. Fibroplasie, Greffon corneen, Osteo-Odonto-Keratoplastik, Silikonimplantate,

Sutures, Tarsal plates, Trabeculotomy

354

Index rerum ad Vol. 176

Microphthalmos, v. Retinoblastoma Microscope électronique par balayage, v. Human retina Microscopie électronique, v. Astrocytoma,

Fabry's disease, Glycogenosis, Human retina,

Hurler/Scheie phenotype, Mitosis Missbildung, v. Aniridia, Congenital

malformations, Neurofibromatosis,

Retinoblastoma Mitosis in the nontumorous parts of the retina

with retinoblastoma 27 Mucoid formation, v. Astrocytoma Mucopolysaccharidose, v.

Hurler/Scheie phenotype Müller'sche Stützzellen, v. Mitosis Muscles oculaires, v. Polyneuritis, Silikonimplantate, Sutures Mykose, v. Dacryocystitis Myopia, v. Fibroplasie, Tilted disc

Nahtmaterial, v. Sutures Nanosomia, v. Aniridia Neovascularization, v. Irisangiographie,

Rétinopathie diabétique Neovascularization; intraretinal neovascularization

271 (V) Nerf optique, v. Astrocytoma, Congenital

malformations, Ischaemie Netherlands Ophthalmological Society.

171st Annual Congress, Amsterdam 1977,

a joint meeting with the Irish

Ophthalmological Society 262-300 (V) Netzhaut, v. Acromegaly, Aniridia, Aphakie,

Congenital malformations, Electrophysiological

studies, Fibroplasie, Glycogenosis,

Heredodystrophie, Human retina, Mitosis,

Rétinopathie diabétique, Siderosis Neural principles in vision 178 (B) Neuritis, v. Polyneuritis

Neurofibromatosis, v. Astrocytoma Neurofibromatosis and congenital glaucoma.

A case report 155 Neuroma; the multiple mucosal neuroma (MMN)

or multiple endocrine neoplasia (MEN type 3)

syndrome 300 (V) Neuro-Ophthalmology, v. Chordom, Eye dipoles,

Polyneuritis Nylon thread; experience gained with intraocular

nylon thread for fixation of artificial

lenses 289 (V) Nystagmus, v. Polyneuritis

Occlusion de la veine centrale, v. Irisangiographie,

Retinal branch vein occlusion Ocular muscles, v. Polyneuritis, Silikonimplantate,

Sutures Ocular pressure, v. Komplikationen Onchocerciasis; some ophthalmic and public 
health aspects of onchocerciasis in the Bawku District (Northern Ghana) 297 (V) Operation, v. Astrocytoma, Fibroplasie, Greffon cornéen, Komplikationen, Silikonimplantate,

Sutures, Tarsal plates Ophthalmoplegie, v. Polyneuritis Optic nerve, v. Astrocytoma, Congenital malformations, Ischaemie Optic neuropathy; acute ischemic optic neuropathy 290 (V) Orbita, v. Chordom, Plasmacytoma, Silikonimplantate ORL, v. Silikonimplantate Os, v. Osteo-Odonto-Keratoplastik, Silikonimplantate Osteo-Odonto-Keratoplastik; Untersuchungen über die Verwendbarkeit von Glaskeramiken zur Osteo-Odonto-Keratoplastik 105 Oxygénothérapie, v. Fibroplasie Panophthalmitis; late panophthalmitis after lens extraction followed by lens implantation 298 (V) Papille, v. Ischaemie, Tilted disc Paralysie du regard, v. Polyneuritis Parese, v. Polyneuritis, Silikonimplantate Paupiere, v. Eryhtrodermia ichthyosiformis, Lidtumoren, Tarsal plates Perforating corneal transplantation - indications and contraindications 275 (V) Perméabilité des membranes, v. Immunopathology Phakomatose, v. Astrocytoma, Neurofibromatosis Photocoagulation, v. Electrophysiological studies, Lightcoagulation, Retinal branch vein occlusion, Rétinopathie diabétique, Trabeculotomy Photogenic retinal lesions and activation of an acute posterior multifocal placoid pigment epitheliopathy 271 (V) Photorezeptoren, v. Human retina Pigmentanomalie, v. Aphakie Pigmentepithel, v. Heredodystrophies, Siderosis, Vitelline dystrophy Pigmentretinopathie, v. Heredodystrophies Index rerum ad Vol. 176 355

Plasmacytoma; orbital involvement of an extramedullary plasmacytoma 241 Plastische Chirurgie, v. Silikonimplantate, Tarsal plates Polyneuritis; die ophthalmoplegische Polyneuritis. Ein klinischer Beitrag zum Fisher-Syndrom 6 Polyposis nasi, v. Plasmacytoma Pompe's disease, v. Glycogenosis Prematures, v. Fibroplasie Proceedings 262-300 (V) Prostaglandins; role of prostaglandins in local inflammation: their significance in ophthalmology 262 (V) Proteine, v. Immunopathology Pupille, v. Polyneuritis Purtscher's disease $273(\mathrm{~V})$

Rabbit, v. Immunopathology, Keratitis sicca, Lid-tumoren, Osteo-Odonto-Keratoplastik, Rejection Raster-Elektronenmikroskop, v. Human retina Reading: the critical influence of timings 296 (V) Recherches expérimentales, v. Immunopathology, Keratitis sicca, Lidtumoren, Osteo-Odonto-Keratoplastik, Rejection, Trabeculotomy Reducing effect on the intraocular pressure of Atenolol 4\% eyedrops during protracted use 263 (V) Refraction, v. Cataract and lens Refraction by computer 294 (V) Rejection; failure of inhibiting corneal graft rejection in rabbits by $5 \alpha$-androstane-3,17-dione 45 Retardation mentale, v. Aniridia Retina, v. Acromegaly, Aniridia, Aphakie, 
Congenital malformations, Electrophysiological studies, Fibroplasie, Glycogenosis, Heredodystrophie, Human retina, Mitosis, Rétinopathie diabétique, Siderosis Retinal branch vein occlusion treated by

photocoagulation 266 (V) Retinal degeneration, v. Aphakie Retinal detachment, v. Aphakie, Fibroplasie Retinal diseases; symposium on retinal

diseases 179 (B) Retinal hole, v. Aphakie Retinoblastoma, v. Mitosis Retinoblastoma in a microphthalmic eye 24 Retinoblastoma combined with severe chromosomal disorders 280 (V)

Retinopathia diabetica, v. Acromegaly,

Electrophysiological studies, Rétinopathie

diabétique Rétinopathie diabétique; la photocoagulation dans

la rétinopathie diabétique 335 Rétinopathie pigmentaire, v. Heredodystrophies Retrolental

fibroplasia, v. Fibroplasie Riesenzellarteriitis, v. Ischaemie Rosacea; treatment of ocular rosacea with oral

tetracycline 300 (V) Rubella optic neuropathy 274 (V) Rubeosis iridis, v. Irisangiographie s $\beta$ Rubidium, v. Immunopathology

Sauerstoffbehandlung, v. Fibroplasie Sauflon 85\% versus Japanese

Menicon 2000288 (V) Scanning electron microscope, v. Human retina Scleromalacia perforans 288 (V) Sehschärfe, v. Keratoconus Side effects of surface anesthetics in

ophthalmological practice 266 (V) Siderosis; histopathological changes in

siderosis bulbi 205 Silikonimplantate; Ergebnisse nichtfixierter

Silikonimplantate in der Versorgung der

Blow-out-Fraktur und Orbitratrümmerfraktur

220 Simultaneous bilateral fluorescein

angiography 272 (V) Sinus maxillaris, v. Chordom, Plasmacytoma,

Silikonimplantate Spaltlampenphotographie der vorderen

Augenabschnitte 179 (B) Steroid, v. Rejection Stoffwechsel, v. Fabry's disease, Glycogenosis, Heredodystrophies, Hurler/Scheie phenotype,

Irisangiographie, Vitelline dystrophy Strabismus in der Praxis 59 (B) Streiff; pour le 70e

anniversaire du Professeur

Bernard Streiff 240 Surdité, v. Aniridia Surgery, v. Astrocytoma, Fibroplasie, Greffon

cornéen, Komplikationen, Silikonimplantate,

Sutures, Tarsal plates Surgery; advances in vitreous surgery 59 (B) Sutures; polyglycolic acid sutures in cataract and

extraocular muscle surgery 102 Synapsen, v. Human retina Syndrom, v. Aniridia, Polyneuritis 356

Index rerum ad Vol. 176

Tapstoretinal dystrophies, v. Heredodystrophies,

Hurler/Scheie phenotype Tarsal plates; repair of defects of the eyelids and

tarsal plates 211 Taubheit, v. Aniridia

Telecommunication in ophthalmology 270 (V) Tension oculaire, v. Komplikationen Therapie, v. Astrocytoma, Keratoconus,

Rétinopathie diabétique Thermokeratoplasty 294 (V) Three-mirror contact lens, v. Aphakie

Tilted disc; X-chromosomal recessive night

blindness and tilted disc anomaly. A case 
report 160 Tolosa-Hunt; the Tolosa-Hunt syndrome 278 (V) Trabeculotomy; experimental trabeculotomy with

a monomode argon laser 121 Tränenwege, v. Dacryocystitis, Lidtumoren Transplantation, v.

Greffon cornéen,

Osteo-Odonto-Keratoplastik, Silikonimplantate,

Tarsal plates Trou rétinien, v. Aphakie Tumor, v. Aniridia, Astrocytoma, Chordom,

Erythrodermia ichthyosiformis, Lidtumoren,

Mitosis, Plasmacytoma, Retinoblastoma Twin, v. Retinoblastoma

Ultrasonography, v. Cataract and lens

Unfall, v. Silikonimplantate

Uveitis, v. Immunopathology, Irisangiographie

Vaisseaux, v. Acromegaly, Amyloidosis,

Congenital malformations, Electrophysiological studies, Fabry's disease, Irisangiographie,

Ischaemie, Rétinopathie diabétique

Varia 60, 180

Varicella; an infrequent ophthalmological

complication of varicella: internal

ophthalmoparesis 281 (V) Vererbung, v. Erythrodermia ichthyosiformis,

Fabry's disease, Heredodystrophies,

Hurler/Scheie phenotype, Neurofibromatosis,

Tilted disc, Vitelline dystrophy Verhandlungsberichte 262-300 (V) Verre de contact, v. Aphakie Vertical motor fusion 291 (V) Vessels, v. Acromegaly, Amyloidosis,

Congenital malformations, Electrophysiological

studies, Fabry's disease, Irisangiographie,

Ischaemie, Rétinopathie diabétique Visual acuity, v. Keratoconus Vitelline dystrophy;

monolateral vitelline

dystrophy with unusual EOG findings 81 Vitrectomy; pars plana vitrectomy 286 (V) Vitreous

body, v. Amyloidosis, Aphakie,

Fibroplasie, Siderosis Voies lacrymales, v. Dacryocystitis, Lidtumoren Vorderkammer, v.

Siderosis

Wachstumshormon, v. Acromegaly Wilms-Tumor, v. Aniridia Winkelblockglaukom, v.

Irisangiographie

Zelleinschlüsse, v. Fabry's disease, Glycogenosis,

Siderosis Zellteilung, v. Mitosis Zentralvenen-Verschluss, v. Irisangiographie,

R. Retinal branch vein occlusion Ziliarkörper

v. Siderosis Zirkulation

v. Irisangiographie Zonular cataract 289 (V) Zwergwuchs

v. Aniridia Zwilling

v. Retinoblastoma Zytologie

v. Keratitis sicca 